\title{
Las presesiones como estrategia para tratar las conductas problemáticas de los alumnos con discapacidad intelectual: una revisión
}

\section{David Simó-Pinatella, Elisabeth Alomar-Kurz Universitat Ramon Llull}

\author{
Joaquima Planella-Morató \\ Universidad de Vic
}

\author{
Josep Font-Roura \\ CPTEstel
}

Climent Giné

Universitat Ramon Llull

\author{
Peter McGill \\ University of Kent (Reino Unido)
}

\section{RESUMEN}

La presesión es una estrategia de intervención sobre los antecedentes que se utiliza para evaluar el impacto de las operaciones motivadoras (OM) sobre el problema de conducta. El presente trabajo revisa aquellos artículos empíricos que han utilizado esta estrategia para tratar la conducta problemática que manifiestan las personas con discapacidad intelectual en edad escolar. Se realizó una búsqueda bibliográfica entre los años 2000 y 2010. Se han encontrado 12 artículos implicando un total de 24 participantes. Los estudios han sido analizados teniendo en cuenta las siguientes variables: características de los participantes, diseño de la investigación, contexto, problema de conducta, función de la conducta, tipología de la OM, presesión y resultados. Los resultados de la revisión sugieren que la presesión tiene un claro efecto sobre la conducta problemática. El artículo finaliza con las implicaciones prácticas que tiene esta estrategia y planteando aquellas cuestiones que los trabajos futuros deberían tener en cuenta.

Palabras clave: Operación motivadora, presesión, problema de conducta, función de la conducta, discapacidad intelectual.

\section{ABSTRACT}

The pre-session is an antecedent intervention strategy that is used to evaluate the impact of motivating operations (MO) on problem behavior. The present paper reviews those empirical studies that have used this strategy to address problem behavior displayed by people with intellectual disabilities at school-age. A systematic literature review was conducted between years 2000 and 2010. A total of 12 published articles representing 24 participants were examined. The studies have been analyzed according to the characteristics of the participants, research design, context, problem behavior, behavioral function, type of the $\mathrm{MO}$, pre-session and results. Findings from this review suggest that a pre-session condition usually has a clear effect on problem behavior. The practical implications of this findings and potential issues for future research are discussed.

Keywords: Motivating operation, pre-session, problem behavior, behavioral function, intellectual disabilities. 
Muchas personas con discapacidad intelectual (DI) manifiestan conductas problemáticas como la destrucción de la propiedad, las agresiones, las rabietas, etc. (Luiselli, 1998) afectando negativamente la calidad de vida de las propias personas (Langthorne, McGill, \& O'Reilly, 2007) y la de sus familiares o profesionales (Emerson, 2001). Las conductas desafiantes se convierten en una de las mayores barreras a las que los sistemas educativos deben dar una respuesta (Font \& Castells, 2009) así como uno de los mayores retos para garantizar la inclusión y participación de las personas con DI en la comunidad (Carr, Ladd, \& Schulte, 2008; McAtee, Carr, \& Schulte, 2004).

Las intervenciones que se centran en la modificación de la conducta, normalmente implican la manipulación (o el control) de determinados eventos con el fin de eliminar, o al menos reducir, dicha conducta (Smith, 2011).

Una manera de tratar las conductas problemáticas es mediante el análisis funcional. Este término, introducido por Skinner en 1953, tenía el objetivo de nombrar el proceso por el cual se podían identificar aquellas variables independientes que están relacionadas de manera funcional con la conducta humana (Betz \& Fisher, 2011). Es decir, permite identificar aquellas variables que están relacionadas funcionalmente con la ocurrencia de la conducta problemática (Miltenberg, 1998); el énfasis se sitúa en la función de la conducta en lugar de en su forma (Iwata, Dorsey, Slifer, Bauman, \& Richman, 1994). Así, el análisis funcional de la conducta se convierte en un método individualizado y comprensivo que posibilita identificar la razón o la función de una con- ducta problemática con el fin de diseñar e implementar intervenciones individualizadas a partir de este conocimiento (Riffel, 2011; Steege \& Watson, 2009). De este modo, se asume una importancia cada vez mayor de los factores ambientales; la conducta no se produce al azar, sino que se manifiesta en un contexto de múltiples variables que interactúan (individuales, de motivación, de instrucción, ambientales y biológicas). Esta complejidad, añade nuevos retos a la comprensión y el tratamiento de las conductas problemáticas.

En los últimos años es posible encontrar distintos trabajos de revisión centrados en la evaluación y el tratamiento de las conductas problemáticas para personas con DI. Por ejemplo, Hanley, Iwata, y McCord (2003) revisaron un total de 277 estudios que llevaban a cabo un análisis funcional e identificaban la relación existente entre los eventos del entorno y las conductas desafiantes. Más recientemente, Heyvaert, Maes, y Onghena (2010) completaron un metaanálisis con el fin de observar los efectos que tenían distintas intervenciones sobre los problemas de conducta. Por otro lado, Lang, Didden et al. (2010) llevaron a cabo una revisión de la literatura centrándose en las intervenciones realizadas para tratar una conducta autolesiva de daños en la piel (skinpicking).

En general, estas revisiones se han diseñado para mostrar la dificultad que los problemas de conducta suponen para los profesionales y sus familias. Además, normalmente se han centrado en la evaluación de los efectos de la evaluación funcional y de los distintos tratamientos o intervenciones (por ej., Brosnan \& Healy, 2011; 
Denis, Van den Noortgate, \& Maes, 2011; Hanley et al., 2003; Heyvaert et al., 2010; Matson \& Neal, 2009).

Actualmente, se puede encontrar un gran volumen de literatura interesada en la comprensión y tratamiento de los problemas de conducta en personas con DI a partir de los antecedentes (por ej., Ahearn, 2003; Butler \& Luiselli, 2007; Garbutt \& Furniss, 2007; Lanovaz, Fletcher, \& Rapp, 2009; Roscoe, Carreau, MacDonald, \& Pence, 2008; Simó-Pinatella, 2008) que son eventos que preceden a los problemas de conducta influenciando su ocurrencia (Miltenberg, 1998). La evidencia demuestra que algunas modificaciones en las variables de antecedentes pueden tener un impacto en el estímulo discriminativo-conducta-consecuencia (three term contingency) (Edrisinha, O'Reilly, Sigafoos, Lancioni, \& Choi, 2011). Es decir, existe una relación dinámica entre los antecedentes, las variables individuales y las consecuencias (Steege \& Watsosn, 2009).

Steege y Watson (2009) exponen que actualmente se puede encontrar dos tipos: los estímulos discriminativos y las operaciones motivadoras. Se entiende que un estímulo discriminativo es aquel que precede la ocurrencia de la conducta y señala la probabilidad de que se mantenga el refuerzo posterior a la conducta (Steege \& Watson, 2009). En consecuencia, es más probable que la conducta se manifieste cuando el antecedente (estímulo discriminativo) está presente en un futuro (Carr et al., 2008; McGill, Teer, Rye, \& Hughes, 2005). A modo de ejemplo, la actividad de demanda ha sido identificada como un indicador potencial para una gran varie- dad de conductas problemáticas (Cale, Carr, Blakeley-Smith, \& Owen-DeSchryver, 2009). Por otro lado, una operación motivadora es un evento que altera (a) la eficacia de un estímulo que actúa como reforzador; y (b) la frecuencia actual de las conductas que han sido reforzadas por ese estímulo (Michael, 2007).

Es decir, las OM son antecedentes que tienen dos propiedades principales (Laraway, Snycerski, Michael, \& Poling, 2003). La primera, el efecto de alterar el valor (value-altering effect), hace referencia al efecto que tiene un antecedente sobre la efectividad de otro estímulo que actúa como un tipo de refuerzo o castigo (Langthorne \& McGill, 2009). Por ejemplo, un caso típico. Una persona con DI manifiesta una conducta problemática (conducta estereotipada) para llamar la atención. Los profesionales no le prestan atención. Esta ausencia aumenta la efectividad reforzadora de la atención que pueda recibir la persona con DI después de realizar la estereotipia; mientras que el valor disminuye una vez ha recibido la atención social deseada.

La segunda, el efecto de alterar la conducta (behavior-altering effect), hace referencia al efecto que tiene de alterar la probabilidad de que se realice una conducta que ha sido asociada a unas consecuencias en el pasado (Laraway et al., 2003). Retomando el mismo ejemplo, dicha persona tendrá más posibilidades de manifestar las conductas que han sido asociadas con recibir atención en el pasado (en este caso, la conducta estereotipada). Así pues, la probabilidad de que la persona realice la misma conducta disminuye después de haber recibido atención. 
Centrándonos en las variables relacionadas con las operaciones motivadoras, se puede observar que en la literatura han sido nombradas de distintas maneras: eventos situacionales (Morris \& Midgley, 1990), variables ecológicas, (O'Neill et al., 1997) o variables contextuales (McAtee et al., 2004). Aunque se entiende que dichas variables han sido analizadas desde distintos marcos teóricos (Kennedy \& Meyer, 1998), a lo largo del presente trabajo nos referiremos a ellas como operaciones motivadoras (OM).

En la literatura, se han reconocido tres categorías principales de OM: biológica, física y social (Carr, Smith, Giacin, Whelan, \& Pancari, 2003). Tal como expone McGill (2011), las OM biológicas incluyen la enfermedad, el dolor o el cansancio entre otros, mientras que las OM físicas incluyen características del entorno como la temperatura, el nivel de ruido, la presencia o ausencia de objetos, etc. Por otro lado, las OM sociales tienen en cuenta las dificultades en la comunicación, actividades que no gustan, falta de opciones para escoger, la atención recibida, etc.

Una manera de analizar el impacto que tienen las OM y, por lo tanto, de explorar aquellas variables que influyen en los problemas de conducta es a partir de las presesiones. A pesar de la gran dificultad que supone encontrar en la literatura una definición de esta estrategia, actualmente se entiende que una presesión supone crear una condición que se proporciona antes de una situación referente en la que la persona puede tener acceso (o una modificación -o deprivación- de acceso) a un evento que mantiene la conducta problemática.

En síntesis, como se ha explicado anteriormente, la evaluación funcional permite enten- der la función de la conducta y, a su vez, detectar aquellas OM que la influencian. Con dicha información, y a partir de las presesiones, se crea una condición experimental que permite evaluar el impacto que tienen dichas variables. Una condición se refiere a unas circunstancias creadas en una fase experimental o un conjunto de sesiones dentro de un experimento (VoIlmer \& Van Camp, 1998).

Por ejemplo, una persona con síndrome de Down realiza una conducta problemática puntual (por ej., autolesionarse). El análisis funcional indica que la función de la conducta es de refuerzo social. Así, utilizando un diseño experimental se crean dos condiciones que se realizan antes de una situación referente (presesiones) para ver el efecto que tiene la OM. En una de las condiciones se facilita atención a la persona que manifiesta la conducta problemática (presesión - acceso a atención social) mientras que en la otra no se le facilita atención (presesión - deprivación/no acceso a atención social). Si la conducta aumenta, se dice que la OM tiene un efecto establecedor (establishing effect) mientras que si la conducta disminuye, el término que se utiliza es efecto de abolición (abolishing effect).

Varios estudios han examinado los efectos de las OM sobre el problema de conducta a través de las presesiones (Chung \& CanellaMalone, 2010). Curiosamente, en nuestro país es difícil encontrar artículos empíricos o de revisión que evalúen dicho efecto en personas con DI. Así, la presente investigación nace, por un lado, ante la necesidad de avanzar en la concepción de las OM que influencian los problemas de conducta de las personas con 
DI y, en concreto, sobre la estrategia de las presesiones. Por otro, por los resultados obtenidos en un estudio previo (Simó-Pinatella et al., en prensa) en el que se revisan aquellas intervenciones sobre las OM que influencian los problemas de conducta de las personas con DI. Los resultados de este estudio de revisión revelan que la estrategia de presesión es una de las más utilizadas en el momento de tratar las conductas problemáticas.

En concreto, el trabajo que se presenta tiene dos objetivos específicos: (a) llevar a cabo una revisión a nivel internacional de aquellos estudios que han utilizado la estrategia de presesión para ver el impacto que tiene la OM sobre la conducta problemática de los participantes con DI en edad escolar $y$, (b) presentar las implicaciones que tiene el uso de las presesiones para tratar los problemas de conducta en nuestra práctica diaria.

\section{Método}

\section{Procedimiento}

La búsqueda bibliográfica del presente estudio de revisión se realizó en las bases de datos PsychInfo, Education Resources Information Center, Science Direct, Blackwell, Sage Publications y Medline (Ebsco y PubMed) entre los años 2000 y 2010. Para localizar los artículos en las distintas bases de datos, se utilizó una combinación de las siguientes palabras clave: «operaciones motivadoras, eventos situacionales, antecedentes, operaciones establecedoras, evaluación del contexto, intervención en el contexto y evaluación funcional» y "problemas de conducta, conducta desafiante, modificación de la conducta, retraso mentaly discapacidad intelectual». Teniendo en cuenta que en este estudio se pretende hacer una revisión de la literatura a nivel internacional, dichos términos fueron escritos en lengua inglesa.

La búsqueda electrónica inicial dio lugar a un total de 353 artículos. El primer, tercer y quinto autor examinaron independientemente los resúmenes de estos 353 artículos con el propósito de seleccionar aquellos que cumplían con los criterios de inclusión (ver siguiente sección). Si los tres autores creían que el artículo cumplía con los criterios de inclusión, el artículo era seleccionado. Si alguno de los autores no estaba seguro, el artículo también se incluía para una futura revisión. Después de distintas reuniones, se seleccionaron un total de 34 artículos que hacían una evaluación y un posterior tratamiento de la conducta problemática a partir de la modificación de los antecedentes (OM). Teniendo en cuenta que durante la búsqueda electrónica se podían haber perdido artículos, se realizó una búsqueda manual del apartado de referencias bibliográficas de estos 34 artículos seleccionados. Aún así, dicha búsqueda no proporcionó más artículos de los ya incluidos en la búsqueda inicial. De estos 34, se rechazaron todos aquellos cuya intervención no utilizaba la estrategia de presesión. Finalmente, se seleccionaron un total de 12 artículos.

\section{Criterio de inclusión y exclusión}

Los artículos incluidos en este estudio debían cumplir con los siguientes criterios de selección: (a) publicados entre 2000 y 2010, (b) escritos en lengua inglesa, (c) haber realizado una evaluación funcional y una intervención 
posterior utilizando la estrategia de presesión con las OM, (d) ser artículos empíricos, (e) haber sido revisado por pares, (f) que los participantes fueran niños y adolescentes (menores de 18 años de edad), y (g) que los participantes tuvieran DI.

De acuerdo con los objetivos del estudio, se han excluido aquellos artículos que aún habiendo exploradoel efecto de la OM sobrela conducta problemática, no han utilizado la estrategia de presesión para hacerlo (por ej., Kuhn, Hardesty, \& Luczynski, 2009; Lanovaz et al., 2009). Así, teniendo en cuenta los criterios de inclusión y exclusión y los acuerdos entre investigadores, este estudio incluye un total de 12 artículos.

\section{Extracción de datos y acuerdo entre investigadores}

Los artículos seleccionados fueron analizados en cuanto a las siguientes variables: (a) las características (edad y discapacidad), (b) el diseño de la investigación, (c) el contexto, (d) el problema de conducta, (e) la función de la conducta, (f) la tipología de la OM, (g) la presesión (condiciones, tiempo de la presesión y de la sesión referente), y (h) los resultados (el efecto establecedor o de abolición).

Dado que en más de un artículo, se realizaba una evaluación e intervención con más de un participante, en aquellos artículos donde un participante era menor de 18 años y otro mayor (por ej., O'Reilly et al., 2006) o un participante con DI y otro sin DI (por ej., McComas, Thompson, \& Johnson, 2003), sólo se extraían los datos correspondientes a los participantes que cumplían con los criterios de inclusión.

\section{Resultados}

Los resultados se exponen en función a las ocho variables anteriormente citadas. La Tabla 1 resume los 12 artículos seleccionados.

\section{Participantes}

Los 12 artículos seleccionados han incluido un total de 24 participantes. La media de edad entre los participantes es de 9,5 años (rango 4-16). Dieciocho de los participantes son niños mientras que seis son niñas. De acuerdo con los criterios de selección, todos los participantes tienen DI. Además, algunos participantes han sido diagnosticados con autismo $(n=12)$, síndrome de Down $(n=3)$ y discapacidades múltiples $(n=3)$. Otras discapacidades asociadas identificadas en los estudios son trastorno desintegrativo infantil $(n=1)$ y discapacidad auditiva y visual $(n=1)$.

\section{Diseño}

El diseño multielemento es el más utilizado entre los estudios seleccionados $(n=8)$ (por ej., Chung \& Canella-Malone, 2010). Dos estudios utilizan un diseño alternante (por ej. Lang et al., 2009) y uno un diseño de reversión (Carter $\&$ Wheeler, 2007). Sólo un estudio utiliza ambos diseños; diseño de reversión y de multielemento (Roantree \& Kennedy, 2006).

\section{Contexto}

El 58,33\% de los artículos seleccionados ( $n$ $=7)$ se han realizado en un entorno educativo. De estos, el $71,42 \%(n=5)$ se han llevado a cabo en escuelas de educación especial, mientras que el $14,28 \%(n=1)$ en escuelas ordina- 
rias. Sólo en uno de los estudios no se indica la tipología de la escuela (Lang et al., 2009).

Otros centros donde se han realizado intervenciones son: centro de día $(n=1)$, centro clínico de análisis de la conducta $(n=1)$ y servicio residencial $(n=1)$. Únicamente en un estudio el proceso se ha realizado en dos entornos distintos (Cater \& Wheeler, 2007). En este caso, la evaluación se realizó en la universidad mientras que la intervención se llevó a cabo en la escuela de educación especial. Finalmente, destaca que tan solo uno de los artículos no especifica el lugar donde se ha realizado el estudio (Roantree \& Kennedy, 2006).

\section{Problema de conducta}

Las conductas problemáticas tratadas en los artículos seleccionados son la agresión ( $n=$ 7), la conducta estereotipada ( $n=5)$, la autolesión $(n=5)$, la conducta destructiva $(n=4)$ y las rabietas $(n=4)$.

Teniendo en cuenta que cada participante puede manifestar más de una conducta problemática, los datos indican que las conductas más tratadas son las agresiones $(n=12)$, la conducta estereotipada $(n=10)$, las rabietas $(n=10)$ y la conducta destructiva $(n=8)$. Sólo cinco participantes muestran conducta autolesiva.

\section{Función de la conducta}

Las cuatro funciones conductuales refuerzo automático $(n=4)$, atención $(n=3)$, evitación o escape $(n=2)$ y acceso a tangible $(n=2)$ han sido investigadas en los distintos artículos. En tres de los artículos, la conducta problemática se manifestaba por funciones múltiples (por ej., McComas et al., 2003).

Entre los participantes, la función de la conducta más común era por refuerzo automático $(n=10)$ y atención $(n=5)$. Las conductas relacionadas por acceso a tangible, evitación o escape y funciones múltiples están presentes en tres participantes respectivamente.

En la mayoría de los estudios, el método utilizado para conocer la función de la conducta problemática ha sido el análisis funcional $(n=$ 10). Sólo en un estudio se ha realizado una evaluación funcional directa (observaciones en el aula) e indirecta (entrevistas) para conocer dicha información (Carter \& Wheeler, 2007). En un único estudio no se indica el proceso realizado para conocer la función de la conducta que manifiesta el participante (Rapp, 2004).

\section{Operación motivadora}

Con el fin de clasificar las OM investigadas en los distintos estudios, el primer y tercer autor clasificaron, independientemente, las distintas condiciones de presesión (ver tabla 1) teniendo en cuenta la tipología de OM propuesta por Carr et al. (2003). Es decir, las condiciones de presesión exploradas en los artículos fueron agrupadas según la categoría social, biológica o física. Los posibles desacuerdos que pudieran surgir, fueron discutidos hasta mutuo consenso.

Como se puede observar en la Tabla 2, un total de seis estudios han explorado la categoría de OM físicas centrándose en la posibilidad de tener (o no) acceso a ítems preferidos o tangibles $(n=5)$ o proporcionando una mejora/enriquecimiento del entorno $(n=1)$ (por ej. Rapp, 


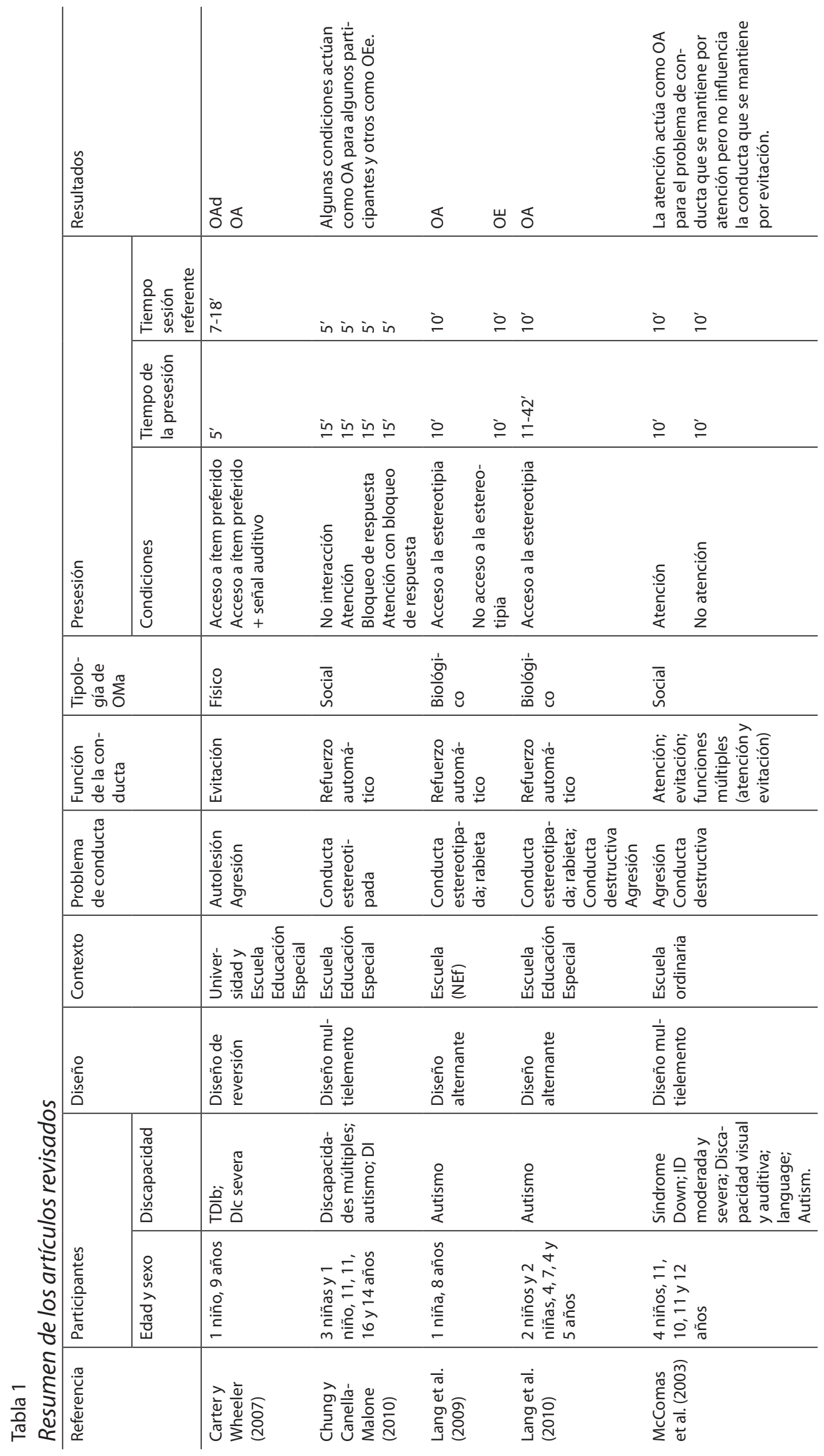




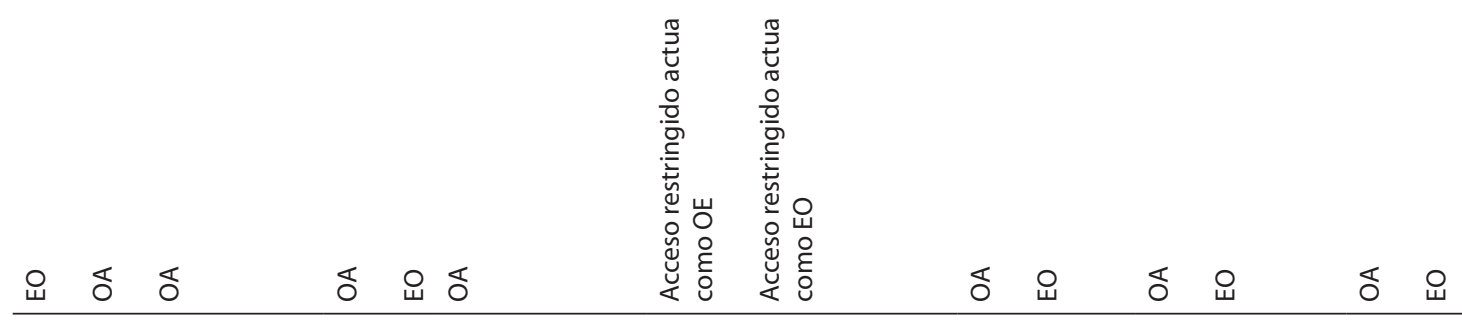

in in in $\div$ 을

\begin{tabular}{|c|c|c|c|c|c|c|c|c|c|c|c|c|c|}
\hline ig & if is & $f$ & in & $\vec{N} \underset{\infty}{\stackrel{i}{n}}$ & $\stackrel{\circ}{-}$ & iे & $\stackrel{\circ}{-}$ & $\dot{m}$ & in & in & iे & іे & ì \\
\hline 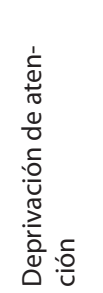 & 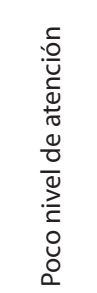 & 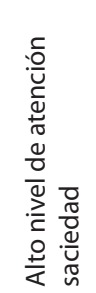 & 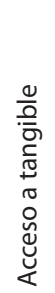 & 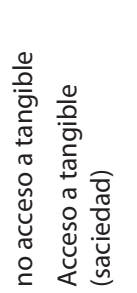 & 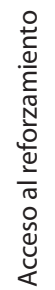 & 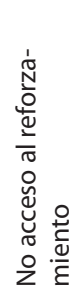 & 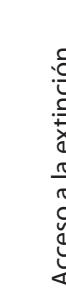 & 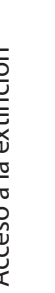 & 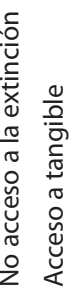 & 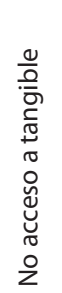 & 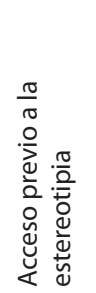 & 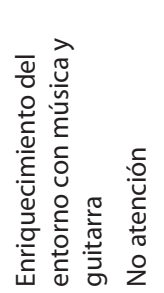 & 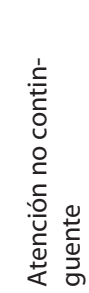 \\
\hline
\end{tabular}

\begin{tabular}{|c|c|c|c|c|c|}
\hline $\begin{array}{l}\overline{\widetilde{\pi}} \\
\overline{0} \\
\stackrel{n}{n}\end{array}$ & 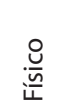 & $\frac{\stackrel{\vartheta}{\underline{M}}}{\underline{\underline{4}}}$ & $\frac{\stackrel{\vartheta}{\underline{M}}}{i \underline{L}}$ & 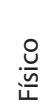 & $\frac{\frac{1}{9}}{\frac{o}{0}} \frac{0}{0}$ \\
\hline
\end{tabular}

\begin{tabular}{|c|c|c|c|}
\hline : & 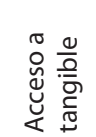 & 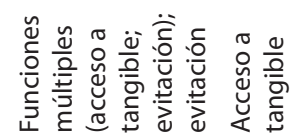 & 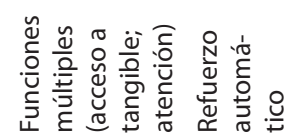 \\
\hline
\end{tabular}

\begin{tabular}{|c|c|c|c|c|c|c|}
\hline 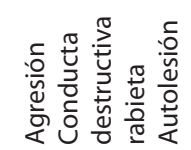 & 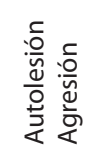 & 兽 & 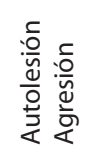 & 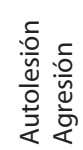 & 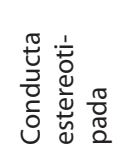 & 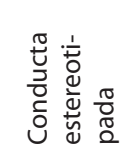 \\
\hline
\end{tabular}

\begin{tabular}{|c|c|c|c|c|c|c|}
\hline 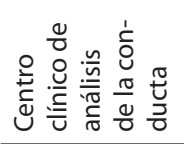 & 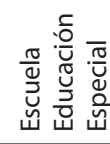 & 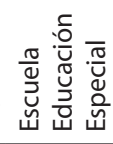 & 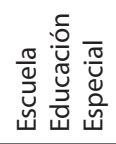 & 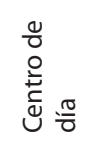 & 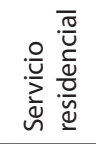 & 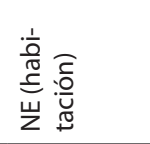 \\
\hline 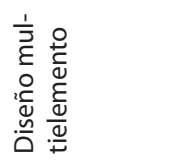 & 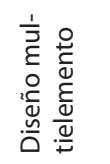 & 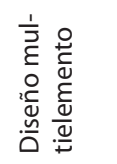 & 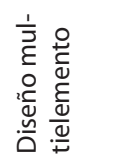 & 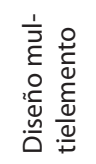 & 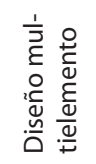 & 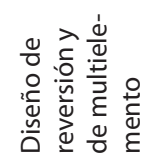 \\
\hline
\end{tabular}

$\underline{\underline{1}}$

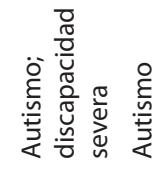

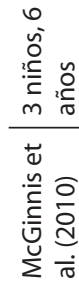

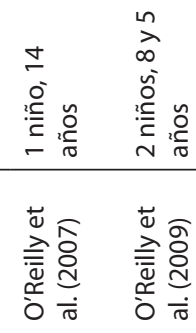

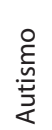

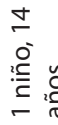

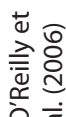

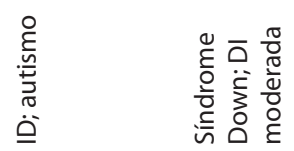

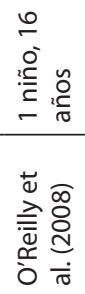

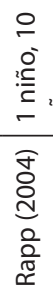

응

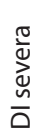

$\overline{0}$ 
2004). Por otro lado, algunos artículos $(n=4)$ se han centrado en la categoría social implicando el nivel de atención proporcionado a los participantes (por ej. McGinnis, Houchims-Juarez, McDaniel, \& Kennedy, 2010). Finalmente, tres estudios se han centrado en la categoría biológica, la cual implica tener (o no) acceso a la conducta problemática (por ej., Rapp, 2004). Resulta necesario comentar que un artículo de estos (O'Reilly et al., 2008) ha observado el valor de dos tipologías de OM distintas (biológica y física).

\section{Presesión}

Con la excepción de dos artículos (Lang, O'Reilly, et al., 2010; O'Reilly et al., 2009) los estudios seleccionados $(n=10)$ han explorado más de una condición de presesión durante la intervención. La mayoría de estos artículos han observado el efecto de la OM a partir de dos (o más) condiciones. Por ejemplo, O'Reilly et al. (2007) estudiaron el valor de la OM para un alumno cuya conducta problemática respondía a la función de acceso a tangible. Para ello, crearon dos condiciones de presesión; en una se facilitaba acceso a tangible antes de la situación referente mientras que en la otra se privaba de dicho acceso. Una característi- ca común entre este estudio y la mayoría de los seleccionados ( $n=8)$ es que la OM ha sido estudiada a partir de dos condiciones de presesión opuestas (por ej. acceso y no acceso a tangible).

Resulta complicado extraer más datos referentes a las condiciones de presesión de la tTabla 1. Aún así, relacionar la función de la conducta con la condición de presesión y el efecto que tiene ésta sobre la conducta problemática, puede tener mucho interés teórico y práctico. Teniendo en cuenta que la mayoría de participantes han sido involucrados en más de una condición de presesión, la Tabla 3 muestra dicha relación por participante.

Esta tabla muestra que algunas funciones de conducta han sido más investigadas que otras. La función de refuerzo automático ha sido explorada a partir de distintas variables (por ej., Rapp, 2004). En concreto se ha explorado a partir de: (a) facilitar acceso (o no) al problema de conducta que manifiestan los participantes, (b) favorecer el entorno en el que se encuentran, y (c) proporcionar distintos niveles de atención. Las conductas problemáticas que se mantienen por atención han sido exploradas en su mayoría de acuerdo con el nivel de atención proporcionado a los participantes (por ej., McComas et al., 2003). Para esta función el

Tabla 2

Tipología de operación motivadora por estudios

\begin{tabular}{ll}
\hline Tipología de operación motivadora & Condiciones de la presesión \\
\hline Físico & - Acceso/no acceso a ítems preferidos $(n=5$ \\
& - Enriquecimiento del entorno $(n=1)$ \\
Social & - Atención/no atención $(n=4)$ \\
Biológico & - Acceso/no acceso a estereotipia $(n=3)$ \\
\hline
\end{tabular}


Tabla 3

Relación entre la función de la conducta, el tipo de la presesión y el efecto de la presesión por participante

\begin{tabular}{|c|c|c|}
\hline Función de la conducta & Tipo de presesión & Efecto de la presesión por participante \\
\hline \multirow[t]{3}{*}{ Evitación o escape } & Acceso a ítem preferido & $\downarrow^{a} \downarrow \downarrow^{* b}$ \\
\hline & Acceso a ítem preferido + señal auditivo & $\downarrow$ \\
\hline & Atención & $={ }^{c}==$ \\
\hline \multirow[t]{7}{*}{ Refuerzo automático } & Acceso al problema de conducta & $\downarrow \downarrow \downarrow \downarrow$ \\
\hline & No acceso al problema de conducta & $\uparrow^{d}$ \\
\hline & Enriquecimiento del entorno & $\uparrow$ \\
\hline & No interacción & $\uparrow \uparrow \downarrow \downarrow$ \\
\hline & Atención & $\uparrow \uparrow \downarrow \downarrow$ \\
\hline & Bloqueo de respuesta & $=\downarrow \downarrow \downarrow$ \\
\hline & Atención con bloqueo de respuesta & $\downarrow==\uparrow$ \\
\hline \multirow[t]{7}{*}{ Atención } & No atención & $\downarrow \uparrow \uparrow \uparrow$ \\
\hline & Atención no contingente & $\uparrow$ \\
\hline & Poco nivel de atención & $\downarrow \downarrow \downarrow$ \\
\hline & Alto nivel de atención & $\downarrow \downarrow \downarrow$ \\
\hline & Atención & $\downarrow \downarrow^{*}$ \\
\hline & Acceso a tangible & $\downarrow^{*}$ \\
\hline & No acceso a tangible & $\uparrow^{*}$ \\
\hline \multirow[t]{3}{*}{ Acceso a tangible } & Acceso a tangible & $\downarrow \downarrow^{*} \downarrow^{*}$ \\
\hline & No acceso & $\uparrow \uparrow *$ \\
\hline & Acceso restringido & $\uparrow$ \\
\hline
\end{tabular}

a Efecto de abolición.

b La conducta de éste participante responde a múltiples funciones.

c Sin efecto.

d Efecto establecedor.

efecto que puede causar tener acceso a tangible es explorado por un participante (O'Reilly et al., 2008). Referente a la función de evitación o escape, las conductas problemáticas se han explorado a partir de las condiciones de presesión focalizadas en acceso a tangible (por ej., Carter \& Wheeler, 2007) y nivel de atención proporcionado (por ej., McComas et al., 2003). Finalmente, la función de acceso a tangible ha sido analizada en los estudios seleccionados a partir de proporcionar (o no) acceso a tangible (por ej., O'Reilly et al., 2006).
Si se retoma la Tabla 1, el tiempo empleado durante las condiciones de presesión oscila entre 5 y 45 minutos. Sólo en un caso la condición llega a durar dos horas (O'Reilly et al., 2007). En este caso se trata de evaluar el efecto que tiene la condición de no acceso a tangible para un participante. Además, y con el objetivo de poder valorar el efecto que tienen dichas condiciones sobre la conducta problemática, el tiempo de la situación referente oscila entre los 5 y 20 minutos. 
Resultados (efecto establecedor o de abolición)

La mayoría de las condiciones de presesión han tenido un efecto (establecedor o de abolición) sobre el problema de conducta que manifiestan los participantes de los estudios seleccionados. Sólo en algunos casos las condiciones de presesión no han tenido ningún efecto sobre la conducta problemática (por ej., McComas et al., 2003). Si se observa el efecto que tiene una condición de presesión sobre la conducta problemática, se puede ver que este efecto puede ser previsible. Por ejemplo, en aquellos estudios cuyo problema de conducta responde a la función de atención (o acceso a tangible), es previsible que facilitar atención (o acceso a tangible) al participante antes de la situación referente reduzca el problema de conducta (por ej., O'Reilly et al., 2009). Es decir, la condición de la presesión tiene un efecto de abolición. Por el contrario, privar a los participantes de dicho acceso, hace aumentar la conducta problemática; actuando así como efecto establecedor (por ej., McGinnis et al., 2010). Aun así, sólo en uno de los participantes cuyo problema de conducta se mantiene por atención, no proporcionar acceso a atención hace disminuir la conducta (Roantree \& Kennedy, 2006). Por otro lado, proporcionar acceso a tangible tiene un efecto de abolición para los problemas de conducta que se mantienen por evitación o escape, mientras que proporcionar atención parece no influenciar dichas conductas. Finalmente, aquellas conductas que se mantienen por refuerzo automático parecen ser reducidas sustancialmente cuando se facilita a los participantes una condición en la que tienen acceso a la conducta (por ej., Lang et al., 2009). Cu- riosamente, el efecto que tiene proporcionar (o no) algún grado de atención a los participantes tiene un efecto mixto (por ej., Chung \& CanellaMalone, 2010). Para algunos de ellos la condición actúa como efecto establecedor mientras que para otros como efecto de abolición. Además, para tres participantes un tipo concreto de atención no influencia el valor de la conducta (Chung \& Canella-Malone, 2010).

\section{Discusión}

Ciertamente, el concepto de operación motivadora está teniendo una importancia significativa en la evaluación y tratamiento de las conductas problemáticas. Las operaciones motivadoras son variables contextuales que alteran la probabilidad de ocurrencia de una determinada conducta ya sea potenciando el efecto evocativo de los estímulos discriminativos y/o el valor de refuerzo de los acontecimientos consecuentes (Laraway et al., 2003; O’Reilly et al., 2008). En los últimos años ha habido un creciente interés en la aplicación de las OM para el tratamiento de las conductas problemáticas (Luiselli, 2006). Asimismo, se empiezan a conocer los efectos de este tipo de intervenciones sobre las conductas problemáticas que presentan las personas con DI (Cale et al., 2009; Carr, Innis, Blakeley-Smith, \& Vasdev, 2004).

El presente trabajo es una de las primeras revisiones que explora el efecto de las presesiones en las conductas problemáticas que presentan los niños y adolescentes con DI.

Como se ha comentado con anterioridad, las presesiones son variables contextuales que funcionan como OM. Así pues, el uso de esta 
estrategia en el tratamiento de las conductas problemáticas debe incluirse en la categoría de la intervención sobre los antecedentes (Luiselli, 2006). Los resultados de este estudio sugieren que el uso de la presesión suele tener un claro efecto sobre la conducta problemática. Es decir, cuando se introduce una situación previa (un acontecimiento antecedente que funciona como OM) a la situación referente en la que se muestra la conducta problemática, se puede observar un efecto establecedor o de abolición sobre dicha conducta.

En los 12 estudios revisados se utilizan diferentes tipologías de presesión. En la mayoría de los casos, la presesión se centra en la posibilidad de tener (o no tener) acceso a un determinado acontecimiento. Este tipo de acontecimientos pueden ser de origen social (por ej. atención), físico (por ej. un tangible) o biológico (por ej. la propia conducta problemática). Es importante destacar que, independientemente a la tipología de la presesión, ésta acostumbra a tener un efecto establecedor o de abolición. Así pues, estos datos parecen indicar que la estrategia de la presesión evalúa el efecto real de la OM. No obstante, el efecto establecedor o de abolición no siempre es el mismo para todos los individuos. A veces la presesión actúa como OM establecedora para unos participantes y de abolición para otros. Este es el caso del estudio de Chung y Canella-Malone (2010), en el que recibir atención sirve de operación establecedora para dos niños y de abolición para otro.

De los 24 participantes, la mayoría presen$\tan$ DI de tipo moderado o severo y autismo. A pesar de ser una población con alto riesgo de conductas problemáticas, sólo un estudio incluye personas con discapacidad múltiple y ninguno con DI de tipo profundo.

Todos los diseños de investigación utilizados en los artículos seleccionados hacen referencia al método experimental que permite evaluar los efectos de una variable independiente (Vollmer \& Van Camp, 1998). Así, los diseños utilizados son el multielemento, el alternante y el de reversión.

La mayor parte de las evaluaciones e intervenciones tienen lugar en situaciones muy controladas como los centros de educación especial o las unidades o servicios especializados. Las conductas problemáticas que se incluyen en los trabajos revisados son la agresión, la conducta estereotipada, la autolesión, la conducta destructiva y las rabietas. Todas ellas consideradas por los profesionales y las familias como las conductas más desafiantes. El refuerzo automático, la atención, la evitación y el acceso a tangible son, por orden de prevalencia, las funciones conductuales que se abordan en los diferentes trabajos analizados. Esto significa que se tratan casi todas las funciones que puede tener la conducta problemática.

En general, la estructura de la presesión de los estudios seleccionados incluye la manipulación de más de una condición (el acceso o no acceso a determinados eventos) durante la intervención. En algunos casos, además, se controla sistemáticamente el tiempo de la presesión.

A partir de estos resultados se pueden extraer algunas conclusiones. En primer lugar, la estrategia de la presesión parece tener un efecto claro sobre la conducta problemática. 
Este efecto puede ser establecedor o de abolición de acuerdo con la condición presente en la presesión. En segundo lugar, esta estrategia pone el acento en los acontecimientos antecedentes de la conducta problemática, permitiendo así plantear un tipo de intervención más ecológico, menos restrictivo e intrusivo. De nuevo, el énfasis en los eventos antecedentes supone un cambio importante en el tratamiento de las conductas problemáticas de las personas con DI, así como la posibilidad de utilizar procedimientos más educativos, preventivos y positivos (Dunlap \& Carr, 2007; Font, 2001; Sailor, Dunlap, Sugai, \& Horner, 2009). En tercer lugar, la importancia de relacionar las intervenciones con la función de la conducta problemática (Ciponi \& Schock, 2007; Steege $\&$ Watson, 2009). En general, los resultados del presente estudio indican que el tratamiento mejora sustancialmente cuando existe una relación clara y definida entre las condiciones que se presentan en la presesión y la función de la conducta. Así pues, se puede deducir que las intervenciones que tienen en cuenta determinadas variables que funcionan como OM, parecen tener un efecto importante en la evaluación y tratamiento de las conductas problemáticas que presentan las personas con DI.

Este trabajo de revisión, no obstante, tiene algunas limitaciones. El reducido número de trabajos no posibilita hacer demasiadas generalizaciones y obliga a ser prudentes en el momento de interpretar los resultados. Por otra parte, centrarse en la población infantil y adolescente no ha permitido incluir los trabajos que se han llevado a cabo en personas adultas con DI. De todos modos, parece adecuado subrayar que disponer de una revisión de estas características y en edad escolar supone una aportación interesante y que puede ayudar al tratamiento de las conductas problemáticas en los centros educativos.

A pesar de estas limitaciones, se pueden enumerar algunas implicaciones prácticas e interrogantes que la investigación futura debería plantearse. En general, la evaluación y las intervenciones sobre las conductas problemáticas exigen un tiempo considerable por parte de los profesionales. La estrategia de la presesión requiere menor tiempo y esfuerzo en relación con otro tipo de tratamientos (Luiselli, 2006; McLaughlin \& Carr, 2005). Además, la presesión supone una estrategia preventiva que evita la manifestación de la conducta problemática y, en consecuencia, el abordaje de situaciones complejas o de difícil manejo. Este tipo de intervención, considerando sus características estructurales, resulta más fácil de aplicar en contextos naturales o en situaciones sociales complejas. Finalmente, conviene subrayar la importancia práctica de combinar de forma adecuada las intervenciones centradas en los acontecimientos antecedentes y en la función de la conducta problemática.

Hay algunas cuestiones que en el futuro la investigación debería cuestionarse. En primer lugar, cómo organizar la presesión para que tenga el mayor impacto posible sobre la conducta problemática. Es necesario definir con más precisión las características estructurales (condiciones presentes, tiempo, etc.) y funcionales de esta estrategia. Por ejemplo, no está claro si el tiempo que dura la presesión puede influenciar el efecto establecedor o de abolición de la OM sobre el problema de conducta (McComas et al., 2003). Asimismo, parece ser que las propiedades 
funcionales de la presesión, más que sus propiedades estructurales, producen un mayor efecto (O'Reilly et al., 2009). Otro aspecto relevante, y que ha recibido poca atención por parte de los investigadores, es la utilidad de diferenciar entre el efecto establecedor y de abolición y el efecto de alterar la conducta que caracteriza las propiedades de las OM (O'Reilly et al., 2007). Es decir, la investigación normalmente se ha centrado en explorar el efecto establecedor y de abolición de las OM (primera propiedad de OM). Aun así, actualmente se dispone de poca investigación orientada a demostrar el efecto evocador y reductor de las OM (segunda propiedad de las OM) (por ej., O'Reilly et al., 2006). Aunque este es realmente un tema complejo, tiene un gran interés teórico y práctico $y$, además, puede facilitar el desarrollo de nuevas y más eficaces estrategias de intervención. Se necesita, también, ampliar las intervenciones sobre los antecedentes a los niños y jóvenes que presentan la discapacidad intelectual más graves (discapacidad profunda y múltiple). Ciertamente, la calidad de vida de estas personas depende fundamentalmente de su bienestar físico y emocional (Petry, Maes, \& Vlaskamp, 2009). Conocer la eficacia de las intervenciones en términos del tiempo en el que se mantiene la mejora, parece ser un tema de preocupación. Finalmente, sería importante que las investigaciones futuras centraran su interés en desarrollar esta estrategia en contextos y situaciones naturales $y$, de esta forma, poder ampliar y generalizar su aplicación.

A fin de cuentas, establecer nuevos enfoques y sistemas para la evaluación y tratamiento de las conductas problemáticas que manifiestan las personas con DI, constituye un reto para los profesionales que se dedican a su atención y educación. Pero básicamente, supone una oportunidad para promover una mejor calidad de vida para esta población.

\section{Referencias}

Ahearn, W. H. (2003). Using simultaneous presentation to increase vegetable consumption in a mildly selective child with autism. Journal of Applied Behavior Analysis, 36, 361-365.

Betz, A. M., \& Fisher, W. W. (2011) Functional Analysis: History and Methods. En Fisher, W. W., Piazza, C. C., \& Roane, H. S., (Eds). Handbook of Applied Behavior Analysis (pp. 206-225). New York: Guilford Press.

Brosnan, J., \& Healy, O. (2011). A review of behavioral intervention for the treatment of aggression in individuals with developmental disabilities. Research in Developmental Disabilities, 32, 437-446.

Butler, L. R., \& Luiselli, J. K. (2007). Escapemaintained problem behavior in a child with autism: Antecedet functional analysis and intervention evaluation of noncontingent escape and instructional fading. Journal of Positive Behavior Interventions, 9, 195-202.

Cale, S., Carr, E., Blakeley-Smith, A., \& OwenDeSchryver, J. S. (2009). Context-based assessment and intervention for problem behavior in children with autism spectrum disorders. Behavior Modification, 33, 707-742.

Carr, E. G., Innis, J., Blakeley-Smith, A., \& Vasdev, S. (2004). Challenging behaviour: research design and measurement issues. En Emerson, E., Hatton, C., Thompson, T., \& Parmenter, T. R. (Eds), The International handbook of applied research in intellectual disabilities, (pp. 423-441). New York: John Wiley \& Sons.

Las referencias marcadas con * indican los artículos incluídos en este estudio de revisión. 
Carr, E. G., Ladd, M. V., \& Schulte, C. F. (2008). Validation of the Contextual Assessment Inventory for problem behavior. Journal of Positive Behavior Interventions 10, 91-104.

Carr, E. G., Smith, C. E., Giacin, T. A., Whelan, B. M., \& Pancari, J. (2003). Menstrual discomfort as a biological setting event for severe problem behavior: Assessment and intervention. American Journal on Mental Retardation, 108, 117-133.

${ }^{*}$ Carter, S. L., \& Wheeler, J. J. (2007). Analysis of behavioural responding across multiple instructional conditions for a child with childhood disintegrative disorder. Journal of Research in Special Educational Needs, 7, 137-141.

Ciponi, E., \& Schock, K. M. (2007). Functional behavioral assessment, diagnosis and treatment. A complete system for education and mental health. New York: Springer Publishing Company.

*Chung, Y. C., \& Cannella-Malone, H. I. (2010). The effects of presession manipulations on automatically maintained challenging behavior and task responding. Behavior Modification, 34, 479-502.

Denis, J., Van den Noortgate, W., \& Maes, B. (2011). Self-injurious behavior in people with profound intellectual disabilities: A meta-analysis of single-case studies. Research in Developmental Disabilities, 32, 911-923.

Dunlap, G., \& Carr, E. G. (2007). Positive behavior support and developmental disabilities: a summary and analysis of research. En S. L. Odom, R. Horner, M. Snell \& J. Blacher (Eds.), Handbook of developmental disabilities. New York: Guilford Press.

Edrisinha, C., O'Reilly, M., Sigafoos, J., Lancioni G., \& Choi, H. Y. (2011). Influence of motivating operations and discriminative stimuli on challenging behavior maintained by positive reinforcement. Research in Developmental Disabilities, 32, 836-845.
Emerson, E. (2001). Challenging behaviour. Analysis and intervention in people with severe intellectual disabilities (2nd ed.). Cambridge: University Press.

Font, J. (2001). El suport conductual positiu: un model d'intervenció pel tractament de les conductes problemàtiques. Suports: Revista Catalana d'Educació Especial, 5, 44-54.

Font, J., \& Castells, M. (2009). El pla educatiu de conducta:el segon nivell d'intervenció peral tractament de conductes problemàtiques. Suports:RevistaCatalanad'EducacióEspecial i Atenció a la Diversitat, 13, 20-27.

Garbutt, N., \& Furniss, F. (2007). Context sampling descriptive assessment: A pilot study of a further approach to functional assessment. Journal of Applied Research in Intellectual Disabilities, 20, 127-130.

Hanley, G. P., Iwata, B. A., \& McCord, B. E. (2003). Functional analysis of problem behavior: $A$ review. Journal of Applied Behavior Analysis, 36(2), 147-185.

Heyvaert, M., Maes, B., \& Onghena, P. (2010). A meta-analysis of intervention effects on challenging behaviour among persons with intellectual disabilities. Journal of Intellectual Disability Research, 54, 634-649.

Iwata, B. A., Dorsey, M. F., Slifer, K. J., Bauman, K. E., \& Richman, G. S. (1994). Toward a functional analysis of self-injury. Journal of Applied Behavior Analysis, 27, 197-209.

Kennedy, C. H., \& Meyer, K. A. (1998). Establishing operations and the motivation of challenging behavior. En J. K. Luiselli, \& M. J. Cameron (Eds.), Antecedent control: Innovative approaches to behavioral support (pp. 329-346). Baltimore: Paul H. Brookes.

Kuhn, D. E., Hardesty, S. L., \& Luczynski, K. (2009). Further evaluation of antecedent social events during functional analysis. Journal of Applied Behavior Analysis, 42, 349-353.

Lang, R., Didden, R., Machalicek, W., Rispoli, M., Sigafoos, J., Lancioni, G., ... Kang, S. (2010). Behavioral treatment of chronic skin- 
picking in individuals with developmental disabilities: A systematic review. Research in Developmental Disabilities, 31,304-315.

*Lang, R., O'Reilly, M., Sigafoos, M., Lancioni, G. E., Machalicek, W., Rispoli, M., \& White, P. (2009). Enhancing the effectiveness of a play intervention by abolishing the reinforcing value of stereotypy: A pilot study. Journal of Applied Behavior Analysis, 42, 889-894.

*Lang, R., O'Reilly, M., Sigafoos, M., Machalicek, W., Rispoli, M., Lancioni, G. E., ... Fragale, C. (2010). The effects of an abolishing operation intervention component on play skills, challenging behavior and stereotypy. Behavior Modification, 34, 267-289.

Langthorne, P., \& McGill, P (2009). A Tutorial on the Concept of the Motivating Operation and its Importance to Application. Behavior Analysis in Practice 2(2), 22-31.

Langthorne, P., McGill, P., \& O'Reilly, M. (2007). Incorporating "motivation" into the functionalanalysisofchallengingbehaviour. On the interactive and integrative potential of the motivating operation. Behavior Modification, 31, 466-487.

Lanovaz, M. J., Fletcher, S. E., \& Rapp, J.T. (2009). Identifying stimuli that alter immediate and subsequent levels of vocal stereotypy: A further analysis of functionally matched stimulation. Behavior Modification, 33, 682-704.

Laraway, S., Snycerski, S., Michael, J., \& Poling, A. (2003). Motivating operations and terms to describe them: Some further refinements. Journal of Applied Behavior Analysis, 36, 407-414.

Luiselli, J. K. (1998). Intervention Conceptualization and Formulation. En Luiselli, J. K. \& Cameron, M. J. (Eds). Antecedent Control. Innovative Approaches to Behavioral Support (pp. 29-44). Baltimore: Paul H. Brookes.

Luiselli, J. K. (2006). Antecedent assessment and intervention. Supporting children and adults with developmental disabilities in community settings. Baltimore: Paul H. Brookes.
Matson, J. L., \& Neal, D. (2009). Psychotropic medication use for challenging behaviors in persons with intellectual disabilities: An overview. Research in Developmental Disabilities, 30, 572-586.

McAtee, M., Carr, E., \& Schulte, C. (2004). A Contextual Assessment Inventory for problem behavior. Journal of Positive Behavior Interventions, 6, 148-165.

McLaughlin, D. M., \& Carr, E. G. (2005). Quality of rapport as a setting event for problem behaviour: Assessment and intervention. Journal of Positive Behavior Interventions, 7, 68-91.

*McComas, J. J., Thompson, A., \& Johnson, L. (2003). The effects of presesion attention on problem behavior maintained by different reinforcers. Journal of Applied Behavior Analysis, 36, 297-307.

McGill, P. (2011). Setting events associated with challenging behaviour: Assessing the realiability, generalisability and validity of the Ecological Interview. Artículo presentado para su publicación.

McGill, P., Teer, K., Rye, L., \& Hughes, D. (2005). Staff reports of setting events associated with challenging behavior. Behavior Modification, 29, 599-615.

*McGinnis, M.A.,Houchins-Juarez, N., McDaniel, J. L., \& Kennedy, C. H. (2010). Abolishing and establishing operation analyses of social attention as positive reinforcement for problem behavior. Journal of Applied Behavior Analysis, 43, 119-123.

Michael, J. (2007). Motivating operations. En J. O. Cooper, T. E. Heron, \& W. L. Heward (Eds.), Applied Behavior Analysis (2nd ed., pp. 374-391). Upper Saddle River, NJ: Pearson Education.

Miltenberg, R. G. (1998). Methods for Assessing Antecedent Influences on Challenging Behaviors. En Luiselli, J. K. \& Cameron, M. J. (Eds), Antecedent Control. Innovative Approaches to Behavioral Support (pp. 4765). Baltimore: Paul H. Brookes. 
Morris, E. K., \& Midgley, B. D. (1990). Some historical and conceptual foundations of ecobehavioral analysis. En S. R. Schroeder (Ed.), Ecobehavioral analysis and developmental disabilities: The 21st century (pp. 1-32). New York: Springer-Verlag.

O'Neill, R. E., Horner, R. H., Albin, R. W., Sprague, J. R., Storey, K., \& Newton, J. S. (1997). Functional assessment and program development for problem behavior: $A$ practical handbook. Pacific Grove, CA: Brooks/Cole Publishing Company.

*O'Reilly, M., Edrisinha, C., Sigafoos, J., Lancioni, G., Cannella, H., Machalicek, W., \& Langathorne, P. (2007). Manipulating the evocative and abative effects of an establishing operation: Influences on challenging behavior during classroom instruction. Behavioral Interventions, 22, 137-145.

*O'Reilly, M. F., Lang, R., Davis T., Rispoli, M., Machalicek, W., Sigafoos, J., ... Didden, R. (2009). A systematic examination of different parameters of presession exposure to tangible stimuli that maintain problem behavior. Journal of Applied Behavior Analysis, 42, 773-783.

*O'Reilly, M. F., Sigafoos, J., Edrisinha, C., Lancioni, G., Cannella, H., Choi, H. Y., \& Barretto, A. (2006). A preliminary examination of the evocative effects of the establishing operation. Journal of Applied Behavior Analysis, 39, 239-242.

*O'Reilly, M. F., Sigafoos, J., Lancioni, G., Rispoli, M., Lang, R., Chan, J., ... Langthorne, P. (2008). Manipulating the behavior-altering effect of the motivating operation: Examination of the influence on challenging behavior during leisure activities. Research in Developmental Disabilities, 29, 333-340.

Petry, K., Maes, B., \& Vlaskamp, C. (2009). Psychometric evaluation of a questionnaire to measure the quality of life of people with profound multiple disabilities (QOL-PMD). Research in Developmental Disabilities, 30, 1394-1405.
*Rapp, J. T. (2004). Effects of prior access and environmental enrichment on stereotypy. Behavioral Interventions, 19, 287-295.

Riffel, L. A. (2011). Positive Behavior Support at the Tertiary Level. California: Corwin.

*Roantree, C., \& Kennedy, C. H. (2006). A paradoxical effect of presession attention on stereotypy: Antecedent attention as an establishing, not an abolishing, operation. Journal of Applied Behavior Analysis, 39, 381-384.

Roscoe, E. M., Carreau, A., MacDonald, J., \& Pence, S. T. (2008). Further evaluation of leisure items in the attention condition of functional analyses. Journal of Applied Behavior Analysis, 41, 351-364.

Sailor, W., Dunlap, G., Sugai, G., \& Horner, R. (2009). Handbook of positive behavior support. New York: Springer.

Simó-Pinatella, D. (2008). Una avaluació funcional per a la millora d'una conducta problemàtica en una alumna amb Síndrome Rett. Suports: Revista Catalana d'Educació Especial i Atenció a la Diversitat, 12, 140-147.

Simó-Pinatella, D., Font-Roura, J., PlanellaMorató, J., McGill, P., Alomar-Kurz, E., \& Giné, C. (2011). Relevance of Types of Motivating Operations in Interventions with Schoolage Children with Intellectual Disabilities and Problem Behavior: A systematic Review. Artículo presentado para su publicación.

Smith, R. G. (2011). Developing Antecedent Interventions for Problem Behavior. En Fisher, W.W., Piazza, C.C., \&Roane, H.S., (Eds). Handbook of Applied Behavior Analysis (pp. 297-316). New York: The Guilford Press.

Steege, M. W., \&Watson, T. S. (2009). Conducting school-based functional behavioral assessment. A practitioner's guide. 2nd edition. New York: Guilford.

Vollmer, T. R. \& Van Camp, C. M. (1998) Experimental Designs to Evaluate Antecedent Control. En Luiselli, J. K. \& Cameron, M. J. (Eds). Antecedent Control. Innovative Approaches to Behavioral Support (pp. 87-100). Baltimore: Paul H. Brookes. 